\title{
Mutual Coupling Effect and Compensation in Non-Uniform Arrays for Direction-of-Arrival Estimation
}

\author{
Elie BouDaher, Fauzia Ahmad", Moeness G. Amin, and Ahmad Hoorfar \\ Center for Advanced Communications, Villanova University, \\ Villanova, PA 19085, USA \\ E-mail: \{eboudahe, fauzia.ahmad, moeness.amin, ahmad.hoorfar\}@ villanova.edu
}

\begin{abstract}
In this paper, we investigate the effect of mutual coupling on direction-of-arrival (DOA) estimation using non-uniform arrays. We compare and contrast the DOA estimation accuracy in the presence of mutual coupling for three different non-uniform array geometries, namely, minimum redundancy arrays (MRAs), nested arrays, and co-prime arrays, and for two antenna types, namely dipole antennas and microstrip antennas. We demonstrate through numerical simulations that the mutual coupling, if unaccounted for, can, in general, lead to performance degradation, with the MRA faring better against mutual coupling than the other two non-uniform structures for both antenna types. We also propose two methods that can compensate for the detrimental effects of mutual coupling, leading to accurate and reliable DOA estimation. Supporting numerical simulation results are provided which show the effectiveness of the proposed compensation methods.
\end{abstract}

Index Terms - Direction finding, mutual coupling, non-uniform arrays.

\footnotetext{
* Corresponding author
} 


\section{INTRODUCTION}

Antenna arrays are employed for direction-of-arrival (DOA) estimation in a broad range of applications including radar, sonar, and wireless communications [1-3]. High-resolution DOA estimation techniques, such as MUSIC [4], ESPRIT [5], and $\ell_{1}-$ SVD [6], are widely used for direction finding. In real antenna arrays, these techniques, in their original implementations, suffer from a model mismatch which, among other factors, can be attributed to mutual coupling between the elements. Mutual coupling occurs when an external illuminating source induces a current on the surface of each array element, causing it to radiate. A portion of the radiated signal is captured by the remaining elements in the array. If unaccounted for, this interaction affects the characteristics and the performance of the array [7-8].

The mutual coupling between the array elements can be captured in a matrix called the mutual coupling matrix (MCM). Two major trends exist in the literature for performing DOA estimation in the presence of mutual coupling. The first deals with the case of perfectly known or modeled MCM, wherein the DOA estimation procedure is modified to account for the coupling [9]. In the second trend, the MCM is assumed to be unknown or imprecisely known with a specific structure, and is jointly estimated along with the source directions.

Electromagnetic theory and numerical or analytical modeling techniques are typically employed to characterize the MCM [8, 10-14]. The MCM depends on the self and mutual impedances between the array elements. One of the earliest methods that model the coupling matrix is the open-circuit method [8]. This method treats the array as a bilateral terminal network and relates the uncoupled voltages with the coupled voltages through a mutual impedance matrix. For dipole antennas, the elements in the mutual impedance matrix can be approximated by closed-form expressions [15]. An extension of the open-circuit method has been proposed in 
[10], where two types of mutual impedances are defined, namely, the transmission mutual impedance and the re-radiation mutual impedance. In [11], the receiving-mutual-impedance method (RMIM) is described for use in receive-only antenna arrays. As such, it provides a more accurate coupling model in DOA estimation applications. RMIM considers each antenna pair separately to compute the receiving mutual impedances. An enhancement of RMIM is presented in [12], which takes into account all the elements simultaneously in order to compute the receiving mutual impedances.

For a perfectly known or modeled MCM, DOA estimation algorithms can be modified to incorporate the coupling and compensate for it in order to achieve accurate source directions [9]. However, if the modeled MCM is not exact, the performance of the DOA estimation is degraded. Moreover, the MCM must be re-calibrated periodically to account for any changes in local conditions. For instance, the presence of a new scatterer in the vicinity of the antenna array changes the mutual coupling. Several methods have been proposed to circumvent these issues. These methods assume the coupling matrix to be unknown or imprecisely known and aim to jointly estimate the MCM along with the source DOAs [7, 16-17]. Ref. [7] presents an iterative method to estimate the MCM, the DOAs, and the antenna gains, wherein the cost function is minimized with respect to one unknown quantity at a time while keeping the remaining two unknowns fixed. A maximum likelihood estimator for DOA estimation under unknown multipath and unknown mutual coupling has been proposed in [16]. Ref. [17] employs sparse reconstruction to perform DOA estimation in the presence of unknown mutual coupling. However, all of these aforementioned methods have been developed for uniform linear arrays (ULAs) and take advantage of the special structure of the corresponding MCMs. Although these methods can be modified and applied to non-uniform arrays, they fail to take advantage of the 
increased degrees-of-freedom (DOFs) offered by non-uniform arrays for DOA estimation [1822]. Recall that an $N_{A}$ - element non-uniform array can provide $O\left(N_{A}^{2}\right)$ DOFs, thereby permitting DOA estimation of more sources than sensors. An iterative method for DOA estimation using non-uniform arrays in the presence of mutual coupling was proposed in [23]. This method treats the non-uniform array as a subset of a ULA and, therefore, cannot take full advantage of the increased DOFs.

In this paper, we investigate the mutual coupling effect in non-uniform arrays. First, we examine the impact of coupling on the DOA estimation accuracy for different array geometries, including minimum redundancy arrays (MRA) [18], nested arrays [20], and co-prime arrays [21, 22]. The performance is evaluated for different array sizes and for two antenna element types, namely, dipole antenna and microstrip antenna. The latter is becoming increasingly popular in radar and wireless communications due to its low profile, ease of fabrication, low cost, and compatibility with radio frequency (RF) circuit boards. A computational electromagnetics software package, FEKO [24], is used to model the antenna arrays, and the RMIM [12] is used to compute the coupling matrices based on the obtained measurements. We show that the MRA provides superior performance compared to the nested and co-prime geometries, irrespective of the antenna type. Second, we propose two compensation methods that allow accurate DOA estimation using non-uniform arrays in the presence of mutual coupling. The first method assumes partial knowledge of the mutual coupling and employs an iterative approach to update the perturbed MCM and DOAs. Sparse signal reconstruction is used to find the source directions for a given coupling matrix, and a global optimization algorithm called covariance matrix adaptation evolution strategy (CMA-ES) [25] is used to update the MCM while keeping the DOAs fixed. The second method assumes unknown coupling and simultaneously estimates the 
MCM, the source powers, and sources directions by minimizing a cost function using CMA-ES. Finally, the effectiveness of the proposed methods is evaluated through numerical examples.

The remainder of this paper is organized as follows. High-resolution DOA estimation using non-uniform arrays is briefly reviewed in Section II. The signal model in the presence of mutual coupling is also presented in the same section. In Section III, DOA estimation performance of different non-uniform array geometries is evaluated and compared for the case of uncompensated mutual coupling. Section IV discusses the two proposed compensation methods that allow accurate DOA estimation under mutual coupling and provides supporting numerical results. Section V concludes the paper.

\section{II.DOA ESTIMATION USING NON-UNIFORM ARRAYS}

A general $N_{A}$ - element linear array is considered. The elements positions are assumed to be integer multiples of the unit spacing, i.e., $x_{i}=n_{i} d_{0}, i=1, \ldots, N_{A}$, where $x_{i}$ is the position of the $i$ th array element, $n_{i}$ is an integer, and $d_{0}$ is the unit spacing which is usually set to halfwavelength at the operating frequency. Assume that $D$ narrowband sources with directions $\left\{\theta_{1}, \theta_{2}, \ldots, \theta_{D}\right\}$ and powers $\left\{\sigma_{1}^{2}, \sigma_{2}^{2}, \ldots, \sigma_{D}^{2}\right\}$ impinge on the array, where $\theta$ is measured relative to broadside. In the absence of mutual coupling, the received data vector at snapshot $t$ can be expressed as

$$
\mathbf{x}(t)=\mathbf{A s}(t)+\mathbf{n}(t)
$$

where $\mathbf{s}(t)$ is the $D \times 1$ source signal vector, $\mathbf{n}(t)$ is the $N_{A} \times 1$ noise vector, and $\mathbf{A}$ is the $N_{A} \times D$ array manifold matrix whose $(i, d)$ th element is given by

$$
[\mathbf{A}]_{i, d}=\exp \left(j k_{0} x_{i} \sin \theta_{d}\right)
$$

Here, $k_{0}$ is the wavenumber at the operating frequency and $\theta_{d}$ is the DOA of the $d$ th source. Under the assumptions of uncorrelated sources and spatially and temporally white noise, the 
covariance matrix can be expressed as

$$
\mathbf{R}_{x x}=E\left\{\mathbf{x}(t) \mathbf{x}(t)^{H}\right\}=\mathbf{A} \mathbf{R}_{s s} \mathbf{A}^{H}+\sigma_{n}^{2} \mathbf{I}
$$

where $E\{\cdot\}$ is the expectation operator, $(\cdot)^{H}$ denotes conjugate transpose, $\mathbf{R}_{s S}=\operatorname{diag}\left\{\sigma_{1}^{2}, \sigma_{2}^{2}, \ldots, \sigma_{D}^{2}\right\}$ is the source covariance matrix, $\sigma_{n}^{2}$ is the noise variance, and $\mathbf{I}$ is an $N_{A} \times N_{A}$ identity matrix.

Two approaches can be used for DOA estimation. The first approach is based on covariance matrix augmentation [26-28], while the second uses spatial smoothing [21-22]. Since the augmented covariance matrix in the first approach may not always be positive semidefinite, we consider spatial smoothing based approach in this paper, which is briefly reviewed below.

Vectorizing the covariance matrix in (3), we obtain

$$
\mathbf{z}=\operatorname{vec}\left\{\mathbf{R}_{x x}\right\}=\widetilde{\mathbf{A}} \mathbf{p}+\sigma_{n}^{2} \tilde{\mathbf{i}}
$$

where $\mathbf{p}=\left[\sigma_{1}^{2}, \sigma_{2}^{2}, \ldots, \sigma_{D}^{2}\right]^{T}$ is the source powers vector, $\widetilde{\mathbf{A}}=\mathbf{A}^{*} \odot \mathbf{A}$, the symbol ' $\odot$ ' denotes the Khatri-Rao product, the superscript ' $*$ ' denotes complex conjugate, and $\tilde{\mathbf{i}}=\operatorname{vec}\{\mathbf{I}\}$ is the vectorized identity matrix. The vector $\mathbf{z}$ emulates measurements at a longer array whose elements positions are given by the difference coarray of the non-uniform arrays, while the $N_{A}^{2} \times D$ matrix $\widetilde{\mathbf{A}}$ is the corresponding manifold matrix [29]. Assuming that the difference coarray has contiguous elements between $-L d_{0}$ and $+L d_{0}$, the data measurements can be rearranged to form a new $(2 L+1) \times 1$ vector $\mathbf{z}_{f}$, which contains measurements at these positions,

$$
\mathbf{z}_{f}=\widetilde{\mathbf{A}}_{f} \mathbf{p}+\sigma_{n}^{2} \tilde{\mathbf{l}}_{f}
$$

Since the sources are replaced by their powers in (5) and the noise is deterministic, the sources now appear as coherent, and subspace-based high-resolution methods can no longer be applied directly to perform DOA estimation. Spatial smoothing is used to build the rank of the 
covariance matrix of $\mathbf{z}_{f}$ [30]. The filled part of the difference coarray is divided into $(L+1)$ overlapping subarrays, each having $(L+1)$ contiguous elements. The positions of the elements of the $m$ th subarray are given by the following set

$$
\left\{(l+1-m) d_{0}, \quad l=0,1, \ldots, L\right\}
$$

The received data vector at the $m$ th subarray is denoted by $\mathbf{z}_{f_{m}}$, and the spatially smoothed covariance matrix is then computed as

$$
\overline{\mathbf{R}}_{z z}=\frac{1}{L+1} \sum_{m=1}^{L+1} \mathbf{z}_{f_{m}} \mathbf{z}_{f_{m}}^{H}
$$

DOA estimation techniques, such as MUSIC, can now be applied to $\overline{\mathbf{R}}_{z z}$ to estimate up to $L$ sources.

Thus far, mutual coupling has been ignored in the signal model. However, in practical antenna arrays, coupling between the antenna elements is a real issue and thus needs to be taken into account. The signal model in (1) can be modified to incorporate mutual coupling as

$$
\mathbf{x}(t)=\mathbf{C A s}(t)+\mathbf{n}(t)
$$

where $\mathbf{C}$ is the $N_{A} \times N_{A}$ mutual coupling matrix. Note that the coupling-free model, discussed in (1), is a particular case of (8) corresponding to $\mathbf{C}$ being an identity matrix. The covariance matrix of the measurements in (8) is given by

$$
\mathbf{R}_{x x}=E\left\{\mathbf{x}(t) \mathbf{x}^{H}(t)\right\}=\mathbf{C A R}_{s S} \mathbf{A}^{H} \mathbf{C}^{H}+\sigma_{n}^{2} \mathbf{I} .
$$

Proceeding with the vectorization and spatial smoothing, followed by DOA estimation without compensating for the MCM, is likely to degrade performance, owing to the mismatch between the assumed model (1) and the actual measurements (8). The severity of performance degradation, however, is a function of the array configuration and the choice of antennas, as shown in the following section. 


\section{III.MUTUAL COUPLING IMPACT ON DOA ESTIMATION}

We quantify the performance degradations due to mutual coupling effect in terms of DOA estimation accuracy for three different non-uniform linear array configurations, namely, the minimum redundancy, nested, and co-prime geometries. Both MRAs and nested arrays have filled difference coarrays, whereas the difference coarray of a co-prime configuration contains a set of the consecutive lags. As such, spatial smoothing based approach can be applied to all three considered configurations for DOA estimation. For comparison, we also provide the performance of a uniform linear array in the presence of mutual coupling.

\section{III.A. Considered Array Geometries}

\section{III.A.1. Uniform Linear Arrays (ULA)}

A uniform linear array is an array whose elements lie along a straight line at equal intervals. An $N_{A}$ - element ULA has elements with positions $\left[0,1, \ldots, N_{A}-1\right] d_{0}$.

\section{III.A.2. Minimum Redundancy Arrays (MRA)}

An MRA maximizes the number of contiguous elements in the difference coarray for a given number $N_{A}$ of antenna elements [18]. The corresponding difference coarray contains the lowest possible redundancy without any missing lags or 'holes'.

\section{III.A.3. Nested Arrays}

An $N_{A}$ - element nested array consists of a combination of two ULAs, where the inter-element spacing of the first ULA with $N_{1}$ elements is equal to the unit spacing $d_{0}$ while the $N_{2}=N_{A}-$ $N_{1}$ elements of the second ULA are separated by an integer multiple of $d_{0}$ [20]. That is, the first element of the second ULA is placed at $\left(N_{1}+1\right) d_{0}$ and the inter-element spacing is also set to 
$\left(N_{1}+1\right) d_{0}$. The corresponding difference coarray is filled and contains no holes.

\section{III.A.4. Co-prime Arrays}

A co-prime array comprises two spatially under-sampled ULAs with co-prime spatial sampling rates $[21,22]$. In the basic co-prime configuration, the first array consists of $M$ elements with inter-element spacing $N d_{0}$ and the second array contains $N$ elements with spacing $M d_{0}$, with $M$ and $N$ being co-prime integers [21]. The elements of the two subarrays are arranged along a single line with the first elements coinciding, resulting in a co-prime array with $N_{A}=M+N-1$ non-uniformly spaced physical elements. The corresponding difference coarray has holes, but is filled between $-(M+N-1) d_{0}$ and $(M+N-1) d_{0}$.

\section{III.B. Mutual Coupling Matrix Modeling and Measurement}

The mutual coupling matrix for each considered non-uniform array configuration is modeled using the receiving-mutual-impedance method (RMIM) [11]. Two conditions must be satisfied in order to render the application of this method feasible [11-12]. First, the array should be in the receiving mode. Second, the antenna elements should be terminated with a known load impedance $Z_{L}$. Assuming these conditions have been fulfilled, the received voltage across the terminal load of a particular antenna can be expressed as a superposition of two external excitations

$$
\mathrm{v}_{i}=Z_{L} \mathrm{i}_{i}=\mathrm{w}_{i}+\tilde{\mathrm{v}}_{i}, i=1,2, \ldots, N_{A}
$$

where $\mathrm{v}_{i}$ is the terminal load voltage of the $i$ th antenna, $\mathrm{i}_{i}$ is the current induced in the $i$ th antenna, $w_{i}$ is the voltage due to the external sources, and $\tilde{v}_{i}$ is the voltage due to the mutual coupling from the other elements in the array. The coupled voltage $\widetilde{v}_{i}$ is given by

$$
\tilde{\mathrm{v}}_{i}=Z_{i, 1} \mathrm{i}_{1}+Z_{i, 2} \mathrm{i}_{2}+\cdots+Z_{i, i-1} \mathrm{i}_{i-1}+Z_{i, i+1} \mathrm{i}_{i+1}+\cdots+Z_{i, N_{A}} \mathrm{i}_{N_{A}},
$$


where $Z_{i, j}$ is the receiving mutual impedance between the $i$ th and $j$ th elements. Substituting (10) in (11) and rearranging, the uncoupled voltages $\mathrm{w}_{i}, i=1,2, \ldots, N_{A}$ can be stacked in a vector $\mathbf{w}$ as

$$
\mathbf{w}=\mathbf{Z v}=\left[\begin{array}{cccc}
1 & -Z_{1,2} / Z_{L} & \cdots & -Z_{1, N_{A}} / Z_{L} \\
-Z_{2,1} / Z_{L} & 1 & \cdots & -Z_{2, N_{A}} / Z_{L} \\
\vdots & \vdots & \ddots & \vdots \\
-Z_{N_{A}, 1} / Z_{L} & -Z_{N_{A}, 2} / Z_{L} & \cdots & 1
\end{array}\right]\left[\begin{array}{c}
\mathrm{v}_{1} \\
\mathrm{v}_{2} \\
\vdots \\
\mathrm{v}_{\mathrm{N}_{\mathrm{A}}}
\end{array}\right]
$$

where $\mathbf{Z}$ is the mutual impedance matrix.

In order to determine the elements of $\mathbf{Z}, K$ plane waves with different DOAs $\left\{\theta_{1}, \ldots, \theta_{K}\right\}$ are individually used to excite the array, and the corresponding received voltages, $\mathrm{v}_{m}^{(k)}, m=$ $1, \ldots, N_{A}, k=1, \ldots, K$, are recorded. Note that $\mathrm{v}_{m}^{(k)}$ denotes the received voltage at the $m$ th array element when the $k$ th plane wave is impinging on the array. The same set of plane waves is also used to excite each array element in isolation in order to measure the uncoupled voltages $\mathrm{w}_{m}^{(k)}$. Given $\mathrm{v}_{m}^{(k)}$ and $\mathrm{w}_{m}^{(k)}$ for all $k$, the following system of linear equations is solved for each antenna element in order to compute the corresponding mutual impedance values.

$$
\left[\begin{array}{c}
\mathrm{v}_{m}^{(1)}-\mathrm{w}_{m}^{(1)} \\
\mathrm{v}_{m}^{(2)}-\mathrm{w}_{m}^{(2)} \\
\vdots \\
\mathrm{v}_{m}^{(K)}-\mathrm{w}_{m}^{(K)}
\end{array}\right]=\left[\begin{array}{cccccc}
\mathrm{v}_{1}^{(1)} & \cdots & \mathrm{v}_{m-1}^{(1)} & \mathrm{v}_{m+1}^{(1)} & \cdots & \mathrm{v}_{N_{A}}^{(1)} \\
\mathrm{v}_{1}^{(2)} & \cdots & \mathrm{v}_{m-1}^{(2)} & \mathrm{v}_{m+1}^{(2)} & \cdots & \mathrm{v}_{N_{A}}^{(2)} \\
\vdots & \ddots & \vdots & \vdots & \ddots & \vdots \\
\mathrm{v}_{1}^{(K)} & \cdots & \mathrm{v}_{m-1}^{(K)} & \mathrm{v}_{m+1}^{(K)} & \cdots & \mathrm{v}_{N_{A}}^{(K)}
\end{array}\right]\left[\begin{array}{c}
Z_{m, 1} \\
\vdots \\
Z_{m, m-1} \\
Z_{m, m+1} \\
\vdots \\
Z_{m, N_{A}}
\end{array}\right]
$$

In order to compute the mutual impedance between each element and the remaining elements in the array, the number of planes waves $K$ should be greater than or equal to $\left(N_{A}-1\right)$ [12]. Once the matrix $\mathbf{Z}$ has been determined, the MCM is computed as $\mathbf{C}=\mathbf{Z}^{-1}$, where $(\cdot)^{-1}$ denotes matrix inverse.

\section{III.C. Performance Comparisons}


In this section, the effect of mutual coupling on the DOA estimation performance is investigated for the array configurations described in Section III.A. We consider two different antenna types, namely, a dipole antenna and a rectangular microstrip or the so-called patch antenna, as array elements. Each antenna is designed for operation at $3 \mathrm{GHz}$. The dipoles are chosen as half-wavelength at $3 \mathrm{GHz}$. Each rectangular patch element has dimensions $L_{p}=$ $31.18 \mathrm{~mm}$ and $W_{p}=46.64 \mathrm{~mm}$, where $L_{p}$ and $W_{p}$ correspond to the resonant length and radiating edge of the patch, respectively. The patch antenna is printed on a $2.87 \mathrm{~mm}$ lossless FR4 substrate with dielectric constant of 2.2, as shown in Fig. 1(a). The ground plane is assumed to be infinite. The patch antenna is modeled using FEKO and the corresponding gain pattern is shown in Fig. 1(b). This antenna is directive with a maximum gain at $\theta=0^{\circ}$ and nulls at $\pm 90^{\circ}$. We assume that the patch elements in the array are positioned with their resonant edges facing each other, as shown in Fig. 1(c) which depicts a six-element uniform linear patch array with an inter-element spacing of half-wavelength at $3 \mathrm{GHz}$.

For each array geometry, we vary the number of elements, $N_{A}$, from four to ten with a step size of two. The element positions of the corresponding MRA configurations are provided in Table I, while those for nested and co-prime geometries are presented in Tables II and III, respectively. Note that, in the case of MRAs, more than one array structure is available for $N_{A}>4$. We choose the configuration which has the least number of element pairs separated by half-wavelength. For co-prime arrays, we consider, for each $N_{A}$, the configuration with $M=$ $N_{A} / 2, N=M+1$. This choice was shown to have operational advantages in [31] and [32]. Further, for nested arrays, we employ the configurations with $N_{1}=N_{2}=N_{A} / 2$; this choice maximizes the DOFs for a given number of antennas [20]. FEKO is used to model the various microstrip and dipole array configurations and measure the required voltages for the RMIM. The 
corresponding mutual impedance and mutual coupling matrices are then computed for the different array geometries with varying number of elements. In the RMIM, the number of plane waves $K$ is set to 16 for all array configurations. The directions of the plane waves are uniformly distributed between $-74^{\circ}$ and $76^{\circ}$.

For each combination of array configuration, antenna type, and total number of elements, we perform 1,000 Monte Carlo runs with two sources at a fixed separation in the reduced angular coordinate, $u=\sin \theta$. That is, for each run, the first source direction $u_{1}$ is randomly chosen to lie between -0.95 and 0.95 and the second source direction $u_{2}$ is selected so that $\Delta u=\left|u_{1}-u_{2}\right|$ is kept constant. Two source separations, $\Delta u=0.1$ and $\Delta u=0.2$, are considered. The model in (8) is used to generate the array measurements, with the signal-to-noise ratio (SNR) set to $0 \mathrm{~dB}$. The total number of snapshots per run is chosen as 10,000 . This high number is selected to remove the influence of i) varying coarray redundancy of different array configurations, and ii) small sample size for correlation matrix estimation as a sample average. Spatial smoothing method is applied in conjunction with MUSIC to estimate the DOAs without compensating for the MCM. Note that in case of co-prime configurations, the DOA estimation only exploits the contiguous part of the coarray. The estimation accuracy is evaluated in terms of the average rootmean-square error (RMSE), which is given by

$$
\overline{R M S E}=\frac{1}{D} \sum_{d=1}^{D} \sqrt{\frac{1}{N_{M C}} \sum_{n=1}^{N_{M C}}\left(\hat{u}_{d, n}-u_{d}\right)^{2}}
$$

where $N_{M C}$ is the total number of Monte Carlo runs and $\hat{u}_{d, n}$ is the estimate of the $d$ th source at the $n$th run.

\section{III.C.1.Dipole Arrays}


Fig. 2(a) depicts the average RMSE as a function of the number of elements for all considered geometries when $\Delta u=0.1$, while the RMSE for $\Delta u=0.2$ is plotted in Fig. 2(c). For reference, the corresponding RMSE plots in the coupling-free scenario are shown in Fig. 2(b) and Fig. 2(d), respectively. Comparing Fig. 2(a) to Fig. 2(b) and Fig. 2(c) to Fig. 2(d), we observe that the results for the coupling-free scenario exhibit much smaller RMSE values than those in the presence of mutual coupling. This confirms the detrimental effect of mutual coupling on the DOA estimation performance. By examining Fig. 2(a) and Fig. 2(c), several additional observations can be made. First, the estimation error decreases as the array size increases for all configurations. Since mutual coupling depends on the distance between the array elements, larger arrays provide a much sparser MCM as compared to smaller arrays, thereby reducing the overall effect on performance. Second, irrespective of the number of elements, the ULA provides the worst performance while the MRA achieves the best performance for both source separations. This is expected because i) the ULA has the highest number of element pairs that are half-wavelength apart, ii) all considered MRAs have a reduced number of antenna pairs separated by half-wavelength, and iii) the MRAs provide both the largest array size for a given number of antennas and largest filled coarray aperture, leading to better resolution capability. Finally, for $\Delta u=0.2$, the co-prime array provides better performance than the nested array for $N_{A}=6,8$, and 10, as seen in Fig. 2(c). This is expected since the nested array has a greater number of element pairs separated by half-wavelength. For $N_{A}=4$, however, the nested array outperforms the co-prime array. This can be explained by examining the two corresponding array structures in Tables II and III. We note that both arrays have three contiguous elements at halfwavelength spacing, while the fourth element is closer to its nearest neighbor in the co-prime array as compared to the nested array. In the case of $\Delta u=0.1$, the roles are reversed for $N_{A}=$ 
6 and 8 , where the nested array outperforms the co-prime array. This is primarily due to the difference in the corresponding resolution capabilities. As mentioned earlier, since the difference coarray corresponding to a co-prime array has holes, a reduced coarray aperture is employed for spatial smoothing based DOA estimation. Even though the coupling effect is larger in nested arrays, its effect on the DOA estimation performance is outweighed by the resolution capability when the sources are closely separated.

\section{III.C.2. Microstrip Arrays}

The Monte Carlo experiments that were performed for dipole arrays are repeated for the microstrip arrays. Fig. 3 shows the obtained average RMSE plots for the different array configurations and different source separations both in the presence and absence of mutual coupling. By comparing the corresponding plots in Fig. 2 and Fig. 3, we observe an increase in the average RMSE when using microstrip arrays. This can be attributed to the proximity of the edges of the consecutive elements. Further, similar to the case of dipole arrays, the microstrip MRA provides the smallest estimation error, while the microstrip ULA has the largest error for both source separations. In addition, the estimation error decreases with increasing number of array elements for all configurations. However, unlike the case of dipole arrays, nested arrays outperform the co-prime arrays for both source separations when microstrip antennas are employed. This performance difference between the co-prime and nested arrays for the two antenna types is due to the fact that mutual coupling in microstrip arrays comprises not only the edge coupling but also the coupling due to the presence of surface waves in the substrate. Since the aperture of co-prime arrays is smaller for the same number of antennas, the surface wave coupling influences the performance of co-prime arrays more than that of nested arrays.

To summarize, mutual coupling affects the DOA estimation performance. The degree of 
performance degradation depends on the array configuration, the number of elements and their types, the source directions, and the source separations.

\section{MUTUAL COUPLING COMPENSATION}

The MCM modeling provides a characterization of the mutual coupling, which can be utilized to account for the coupling in DOA estimation methods. However, in practice, the model can suffer from inaccuracies and, as such, requires frequent re-calibration in order to account for any changes in local conditions. Maintaining an exact MCM model can be cumbersome, if not impossible, in many practical applications. In this section, we propose two compensation methods for accurate DOA estimation under unknown or imperfectly known MCMs. The first method treats the modeling imperfections as perturbations in the MCM and employs an iterative approach to estimate the source directions and the perturbed MCM. The second method performs joint estimation of the MCM and the source directions simultaneously.

\section{IV.A. Iterative Approach}

We model imperfections in the coupling matrix as arising from perturbations in the mutual impedance matrix, i.e., $\mathbf{Z}_{\text {actual }}=\mathbf{Z}_{\text {model }}+\Delta \mathbf{Z}$, where $\mathbf{Z}_{\text {actual }}$ is the actual mutual impedance matrix, $\mathbf{Z}_{\text {model }}$ is the initial modeled mutual impedance matrix, and $\Delta \mathbf{Z}$ is the perturbation matrix. We assume that the sources are sparse in angle, which is true in general for direction finding applications. The angular region of interest is discretized into a finite set of $Q$ grid points, where $Q \gg D$. Substituting $\left(\mathbf{Z}_{\text {model }}+\Delta \mathbf{Z}\right)^{-1}$ for $\mathbf{C}$ in (9) and vectorizing $\mathbf{R}_{x x}$ yields

$$
\begin{aligned}
& \operatorname{vec}\left\{\mathbf{R}_{x x}\right\}=\operatorname{vec}\left\{\mathbf{C A R}_{s s} \mathbf{A}^{H} \mathbf{C}^{H}+\sigma_{n}^{2} \mathbf{I}\right\} \\
& \quad=\left\{\left[\left(\mathbf{Z}_{\text {model }}+\Delta \mathbf{Z}\right)^{-1} \mathbf{A}\right]^{*} \otimes\left[\left(\mathbf{Z}_{\text {model }}+\Delta \mathbf{Z}\right)^{-1} \mathbf{A}\right]\right\} \operatorname{vec}\left\{\mathbf{R}_{s s}\right\}+\sigma_{n}^{2} \tilde{\mathbf{I}},
\end{aligned}
$$

where ' $\otimes$ ' denotes the Kronecker product. In order to solve for the unknowns, namely the perturbations $\Delta \mathbf{Z}$, source directions and powers, and noise variance, a nested optimization 
problem can be posed as

$$
\begin{aligned}
\min _{\Delta \mathbf{Z}} \min _{\operatorname{diag}\left(\overline{\mathbf{R}}_{s s}\right), \sigma_{n}^{2}} & \| \operatorname{vec}\left\{\widehat{\mathbf{R}}_{x x}\right\} \\
& -\left\{\left[\left(\mathbf{Z}_{\text {model }}+\Delta \mathbf{Z}\right)^{-1} \overline{\mathbf{A}}\right]^{*} \otimes\left[\left(\mathbf{Z}_{\text {model }}+\Delta \mathbf{Z}\right)^{-1} \overline{\mathbf{A}}\right]\right\} \operatorname{vec}\left\{\overline{\mathbf{R}}_{s s}\right\}-\sigma_{n}^{2} \tilde{\mathbf{i}} \|_{2} \\
& +\lambda\left\|\operatorname{diag}\left(\overline{\mathbf{R}}_{s s}\right)\right\|_{1}
\end{aligned}
$$

where $\widehat{\mathbf{R}}_{x x}$ is the covariance matrix obtained as a sample average, $\overline{\mathbf{A}}$ is the $N_{A} \times Q$ array manifold matrix corresponding to the grid of potential directions, $\overline{\mathbf{R}}_{S S}$ is the covariance matrix of the potential sources, and $\lambda$ is a Lagrange-type regularization parameter. The elements on the main diagonal of $\overline{\mathbf{R}}_{s s}$ are the powers of the potential sources. The $D$ nonzero diagonal elements correspond to the powers of the actual sources.

The inner optimization in (16) over $\operatorname{diag}\left(\overline{\mathbf{R}}_{s S}\right)$ and $\sigma_{n}^{2}$ is convex and can be solved using sparse reconstruction techniques with the constraint that the unknowns are nonnegative. The $\ell_{2}-$ norm term in (16) is the least squares cost function that maintains data fidelity, while the $\ell_{1}-$ norm term encourages sparsity in the reconstructed power spectrum. The weight of the sparsity constraint in the overall cost function is controlled by $\lambda$. The choice of $\lambda$ is tied to the source sparsity and the noise variance. Several methods have been proposed to estimate $\lambda$ including cross-validation [33] and the discrepancy principle [6]. It is noted that the source directions are assumed to be located on the grid. However, several methods can be used to modify the model in order to deal with off-grid sources $[6,34]$. The outer minimization over $\Delta \mathbf{Z}$ in (16) is non-convex and can be solved by general nonlinear optimization methods. The nested optimization in (16) is solved iteratively until the maximum number of iterations is reached or until the cost function stagnates.

In this paper, we solve the outer optimization problem in (16) using CMA-ES [25], which is a nature-based global optimization algorithm. Nature-based optimization algorithms try to emulate natural phenomena, such as swarm intelligence and the Darwinian model of natural 
evolution, in order to find optimal solutions. These algorithms can deal with highly nonlinear cost functions, which require simultaneous optimization of a large number of parameters. Nature-based optimization algorithms include many categories, such as Genetic Algorithms [35], Particle Swarm Optimization [36], Evolutionary Programming [37], and Evolution Strategies [38]. CMA-ES has been shown to outperform other evolutionary algorithms in many complex electromagnetic problems [39].

CMA-ES is a self-adaptive evolution strategy which requires no parameter tuning. Fig. 4 shows the block diagram of the main operation of CMA-ES. The algorithm starts by initializing the parameters to their default values. It then samples a new generation of potential solutions from a multivariate Gaussian distribution using

$$
\mathbf{y}_{i}^{(g+1)} \sim N\left(\mathbf{m}^{(g)},\left(\sigma_{c}^{(g)}\right)^{2} \mathbf{C}_{c}^{(g)}\right)
$$

where $\mathbf{y}_{i}^{(g+1)}$ consists of the parameters of the $i$ th potential solution at the $(g+1)$ th generation, $\mathbf{m}^{(g)}$ is the mean parameter vector of the best performing members of the previous generation, $\sigma_{c}^{(g)}$ is the step size, and $\mathbf{C}_{c}^{(g)}$ is the covariance matrix of the parameters. The parameters of the multivariate Gaussian distribution are then updated sequentially using the best performing members of the generation [25]. The performance of the members is measured by their fitness value or score on the outer optimization in (16). This process is then repeated until a termination criterion is met. This criterion can be, for instance, a target fitness value or a maximum number of generations.

\section{IV.B. Simultaneous Approach}


In this approach, the sources directions and the MCM are simultaneously estimated rather than in an iterative fashion [40]. Starting with the covariance matrix in (9), the joint DOA and MCM estimation is achieved by solving

$$
\min _{\boldsymbol{\theta}, \operatorname{diag}\left(\mathbf{R}_{s S}\right), \mathbf{z}, \sigma_{n}^{2}}\left\|\widehat{\mathbf{R}}_{x x}-\mathbf{C A} \mathbf{R}_{s s} \mathbf{A}^{H} \mathbf{C}^{H}-\sigma_{n}^{2} \mathbf{I}\right\|_{F}^{2},
$$

where $\|\cdot\|_{F}$ is the Frobenius norm, $\boldsymbol{\theta}=\left[\theta_{1}, \theta_{2}, \ldots, \theta_{D}\right]^{T}$ contains the source DOAs, $\operatorname{diag}\left(\mathbf{R}_{s S}\right)$ consists of the source powers, and $\mathbf{z}$ holds the unique elements in $\mathbf{C}^{-1}$. The total number of unknowns is $(2 D+1+2|\mathbf{z}|)$, where $|\mathbf{z}|$ is the number of unique elements in the mutual impedance matrix. The multiplier 2 in front of $|\mathbf{z}|$ is due to the entries of $\mathbf{z}$ being complex valued. A mixed-parameter variation of CMA-ES is used to solve (18), since the sources DOAs are picked from a predetermined grid while the remaining unknowns are assumed to be continuous parameters $[39,41]$.

It should be noted that the perturbed mutual impedance matrix model can be employed in (18), with the minimization carried out with respect to $\boldsymbol{\theta}, \operatorname{diag}\left(\mathbf{R}_{s S}\right), \sigma_{n}^{2}$, and $\Delta \mathbf{Z}$.

\section{IV.C. Supporting Results}

In the first example, a dipole array with a six-element nested configuration is considered. The elements positions are given by $[1,2,3,4,8,12] d_{0}$. The corresponding difference coarray extends from $-11 d_{0}$ to $11 d_{0}$ and is filled with no holes. The length of the dipoles is set to halfwavelength. The corresponding MCM is modeled using the RMIM and the signal model in (8) is used to generate the array measurements. The coupling matrix is then assumed to be unknown and is jointly estimated along with the DOAs using the simultaneous method. A total of 11 sources are considered. The sources are uniformly spaced between -0.85 and 0.8 in the reduced angular coordinate $u=\sin \theta$. Spatially and temporally white Gaussian noise is added to the 
observations, and the SNR is set to $10 \mathrm{~dB}$. The total number of snapshots is fixed to 1,000 . Mixed-parameter CMA-ES is used to minimize the cost function in (18), where the DOAs are assumed to fall on a grid with $1^{\circ}$ step size and the remaining parameters are assumed to be continuous. The search space for the unknown mutual impedance matrix is restricted to be within $10 \%$ of the actual values. For the CMA-ES algorithm, the population size and the number of generations are each set to 1,000. Fig. 5 shows the estimated spectrum, with the vertical dashed lines indicating the true source locations. Clearly, the proposed method is successfully able to compensate for the mutual coupling and estimate the correct source directions.

Next, we consider $D=5$ sources with directions $\left[-58^{\circ},-26^{\circ},-1^{\circ}, 23^{\circ}, 53^{\circ}\right]$. The same sixelement nested array configuration from the first example is employed to estimate the DOAs under varying number of snapshots and SNR values. Figs. 6(a) shows the success rate as a function of the number of snapshots with the SNR of all sources fixed at $10 \mathrm{~dB}$. For each snapshot value, a total of 100 Monte Carlo runs are used. A solution is deemed successful if each DOA estimate is within $2^{\circ}$ of the true DOA. Fig. 6(b) shows the average RMSE values corresponding to the successful solutions. Figs. 6(c) and 6(d) depict the success rate and the average RMSE as a function of SNR, respectively, with the number of snapshots fixed at 1,000. Again, 100 Monte Carlo runs are employed for each SNR value. From Fig. 6, it is evident that the performance of the proposed method improves with an increasing number of snapshots and increasing SNR. In addition, compared to the SNR, the number of snapshots has a larger effect on the performance. This is expected since the proposed method relies on a good estimate of the covariance matrix using the sample average. It should be noted that the performance can be further improved by increasing the population size and the number of generations of the CMAES algorithm. 
In the third example, the iterative method is used to estimate the actual MCM along with the DOAs. A six-element microstrip array with an MRA configuration is used. The elements positions are given by $[0,1,6,9,11,13] d_{0}$, and each microstrip element is similar to the one modeled in Section III.C. The MCM is modeled using the RMIM and the data measurements are generated using the model in (8). The MCM is then perturbed to emulate the effect of changes in local conditions. The perturbations are drawn from uniform distributions that assume values between $-25 \%$ and $25 \%$ of the actual values. Eight sources, uniformly spaced between $u=-0.7$ and $u=0.6$, are considered. The SNR of all sources is set to $10 \mathrm{~dB}$ and the number of snapshots is equal to 1,000. Fig. 7(a) shows the estimated spectrum using MUSIC with spatial smoothing without accounting for mutual coupling. Clearly, the estimation performance is severely degraded since the mutual coupling is not accounted for. Fig. 7(b) depicts the initial estimated spectrum, while Figs. 7(c) and 7(d) show the estimated spectra after the first and tenth iterations, respectively. The initial estimated spectrum of Fig. 7(b) is based on solving the inner optimization problem in (16) with the perturbations set to zero. We observe that the initial spectrum completely misses one source, provides biased estimates for some sources, and exhibits spurious peaks. The estimated spectrum after the first iteration in Fig. 7(c) finds all the sources, but exhibits some spurious peaks. The performance of the method improves with increasing number of iterations, and after ten iterations, all the sources are correctly estimated, as shown in Fig. 7(d).

In order to validate the convergence of the proposed method, we perform 100 Monte Carlo runs with the aforementioned six-element microstrip MRA. The same source directions and powers as in the third example are considered. In each run, a new perturbation of the MCM is generated and the minimization is performed over 20 iterations. Fig. 8 shows the success rate as 
a function of the iteration number. We note that the success rate improves with an increasing number of iterations and reaches $100 \%$ after 20 iterations. It is to be noted that a faster convergence can be reached for smaller perturbations of the MCM.

In the final example, a six-element extended co-prime dipole array with $M=2$ and $N=3$ is considered. Note that an extended co-prime configuration comprises of two interleaved ULAs, one with $2 M$ elements and the other with $N$ elements, with the first element shared between them. The first subarray has elements at $[0,3,6,9] d_{0}$ and the second one has elements at $[0,2,4] d_{0}$. The corresponding difference coarray is filled between $-7 d_{0}$ and $7 d_{0}$. The simultaneous compensation method is used to jointly estimate the MCM and the DOAs. A total of seven uniformly spaced sources between $u=-0.8$ and $u=0.8$ are considered. The SNR is fixed to $10 \mathrm{~dB}$ for all sources and the number of snapshots is set to 1,000 for each run. Mixedparameter CMA-ES with 1,000 population size and 1,000 generations is used to minimize the cost function. 100 Monte Carlo runs are performed to assess the ability of the proposed method to provide a unique solution. Fig. 9 shows the estimated spectrum of one of the successful runs. The DOA estimates of all 100 runs are superimposed in Fig. 10(a). It is evident that some of the runs result in wrong or biased estimates. The success rate as a function of the maximum bias of all estimates is plotted in Fig. 10(b). For instance, 76 percent of the runs result in a solution that has each estimated DOA within $2^{\circ}$ of the actual value. The success rate can be improved by increasing the number of generations used in CMA-ES. This is validated by increasing the number of generations to 5,000 and introducing restarts after each 1,000 generations. Fig. 11 shows the corresponding results. We observe that runs in excess of 60 percent result in unbiased estimates, while all 100 runs produce solutions having each source estimate within $2^{\circ}$ of the actual value. 


\section{CONCLUSION}

The impact of mutual coupling on DOA estimation performance using non-uniform arrays was investigated in this paper. Direction finding accuracy was compared for three different nonuniform array configurations and two antenna element types. The MRA configuration was shown to provide superior estimation performance compared to nested and co-prime array configurations. Further, choice of dipole antennas as array elements fared better in terms of RMSE over microstrip antennas; the latter suffer from additional coupling arising from surface waves in the substrate. Additionally, two mutual coupling compensation methods were proposed for non-uniform arrays. The first method is iterative in nature and assumes imprecisely known MCM. The second method simultaneously estimates the coupling matrix and the DOAs and is better suited to scenarios where no prior knowledge of the MCM is available. Numerical examples were used to demonstrate the effectiveness of the proposed compensation methods.

\section{ACKNOWLEDGMENT}

This work was supported by the US Office of Naval Research under grant N00014-13-1-0061.

\section{REFERENCES}

[1] S. Chandran, Advances in Direction-of-Arrival Estimation, Norwood, MA: Artech House, 2006.

[2] H.L. Van Trees, Optimum Array Processing: Part IV of Detection, Estimation and Modulation Theory, New York, NY: Wiley, 2002.

[3] T.E. Tuncer and B. Friedlander, Classical and Modern Direction-of-Arrival Estimation, Boston, MA: Academic Press (Elsevier), 2009.

[4] R. Schmidt, "Multiple emitter location and signal parameter estimation," IEEE Trans. Antennas Propag., vol. 34, no. 3, pp. 276-280, 1986.

[5] R. Roy and T. Kailath, "ESPRIT-Estimation of signal parameters via rotational invariance techniques," IEEE Trans. Acoust., Speech, Signal Processing, vol. 37, no. 7, pp. 984-995, 1989. 
[6] D. Malioutov, M. Cetin, and A. Willsky, "Sparse signal reconstruction perspective for source localization with sensor arrays," IEEE Trans. Signal Process., vol. 53, no. 8, pp. 3010-3022, 2005.

[7] B. Friedlander and A.J. Weiss, "Direction finding in the presence of mutual coupling," IEEE Trans. Antennas Propag., vol. 39, no. 3, pp. 273-284, 1991.

[8] I.J. Gupta and A.A. Ksienski, "Effect of mutual coupling on the performance of adaptive arrays," IEEE Trans. Antennas Propag., vol. 31, no. 5, pp. 785-791, 1983.

[9] C.-C. Yeh, M.-L. Leou, and D.R. Ucci, "Bearing estimations with mutual coupling present," IEEE Trans. Antennas Propag., vol. 37, no. 10, pp. 1332-1335, 1989.

[10] H. Yamada, Y. Ogawa, and Y. Yamaguchi, "Mutual impedance of receiving array and calibration matrix for high-resolution DOA estimation," in Proc. IEEE/ACES International Conference on Wireless Communications and Applied Computational Electromagnetics, Honolulu, HI, 2005, pp. 361-364.

[11] H.T. Hui, "Improved compensation for the mutual coupling effect in a dipole array for direction finding," IEEE Trans. Antennas Propag., vol. 51, no. 9, pp. 2498-2503, 2003.

[12] H.S. Lui and H.T. Hui, "Effective mutual coupling compensation for direction-of-arrival estimation using a new, accurate determination method for the receiving mutual impedance," J. of Electromagn. Waves and Appl., vol. 24, pp. 271-281, 2010.

[13] R.S. Adve and T.K. Sarkar, "Compensation for the effects of mutual coupling on direct data domain adaptive algorithms," IEEE Trans. Antennas Propag., vol. 48, no. 1, pp. 8694, Jan. 2000.

[14] H. Singh, H.L. Sneha, and R.M. Jha, "Mutual coupling in phased arrays: A review," International J. Antennas Propag., vol. 2013, Article ID 348123, 2013.

[15] C. A. Balanis, Antenna Theory: Analysis and Design, 3rd Ed, New York: John Wiley and Sons, 2005.

[16] Q. Bao, C.C. Ko, and W. Zhi, "DOA estimation under unknown mutual coupling and multipath”, IEEE Trans. Aerosp. Electron. Syst., vol. 41, no. 2, pp. 565-573, 2005.

[17] J. Dai, D. Zhao, and X. Ji, "A sparse representation method for DOA estimation with unknown mutual coupling," IEEE Antennas Wireless Propagat. Lett., vol. 11, pp. 1210$1213,2012$.

[18] A. Moffet, "Minimum-redundancy linear arrays," IEEE Trans. Antennas Propag., vol. AP-16, no. 2, pp. 172-175, Mar. 1968.

[19] G.S. Bloom and S.W. Golomb, "Application of numbered undirected graphs," Proc. IEEE, vol. 65, no. 4, pp. 562-570, Apr. 1977. 
[20] P. Pal and P.P. Vaidyanathan, "Nested arrays: a novel approach to array processing with enhanced degrees of freedom," IEEE Trans. Signal Process., vol. 58, no. 8, pp. 41674181, Aug. 2010.

[21] P. P. Vaidyanathan and P. Pal, "Sparse sensing with co-prime samplers and arrays," IEEE Trans. Signal Process., vol. 59, no. 2, pp. 573-586, 2011.

[22] P. Pal and P. P. Vaidyanathan, "Coprime sampling and the music algorithm," in Proc. IEEE Digital Signal Processing Workshop and IEEE Signal Processing Education Workshop, Sedona, AZ, 2011, pp. 289-294.

[23] J. Dai, D. Zhao, and Z. Ye, "DOA estimation and self-calibration algorithm for nonuniform linear array," in Proc. International Symposium on Intelligent Signal Processing and Communications Systems, Chengdu, China, 2010.

[24] FEKO Suite 6.3, EM Software \& Systems - S.A. (Pty) Ltd.

[25] N. Hansen and A. Ostermeier, "Completely derandomized self-adaptation in evolutionary strategies," Evolutionary Computation, vol. 9, no. 2, pp. 159-195, 2001.

[26] S.U. Pillai, Y. Bar-Ness, and F. Haber, "A new approach to array geometry for improved spatial spectrum estimation," Proc. IEEE, vol. 73, pp. 1522-1524, Oct. 1985.

[27] Y.I. Abramovich, D.A. Gray, A. Y. Gorokhov, and N. K. Spencer, "Positive-definite Toeplitz completion in DOA estimation for nonuniform linear antenna arrays. I. Fully augmentable arrays," IEEE Trans. Signal Process., vol. 46, pp. 2458-2471, Sep. 1998.

[28] Y.I. Abramovich, N.K. Spencer, and A. Y. Gorokhov, "Positive-definite Toeplitz completion in DOA estimation for nonuniform linear antenna arrays. II. Partially augmentable arrays," IEEE Trans. Signal Process., vol. 47, pp. 1502-1521, Jun. 1999.

[29] R.T. Hoctor and S.A. Kassam, "The unifying role of the coarray in aperture synthesis for coherent and incoherent imaging," Proc. IEEE, vol. 78, no. 4, pp. 735-752, Apr. 1990.

[30] T.J. Shan, M. Wax, and T. Kailath, "On spatial smoothing for direction-of-arrival estimation of coherent signals," IEEE Trans. Acoust., Speech, Signal Process., vol. 33, no. 4, pp. 806-811, 1985.

[31] K. Adhikari, J.R. Buck, and K.E. Wage, "Beamforming with extended co-prime sensor arrays," in Proc. IEEE International Conference on Acoustics, Speech, and Signal Processing, Vancouver, BC, May 2013, pp. 4183-4186.

[32] E. BouDaher, Y. Jia, F. Ahmad, and M. G. Amin, "Multi-frequency co-prime arrays for high-resolution direction-of-arrival estimation," IEEE Trans. Signal Process., vol. 63, no. 14, pp. 3797-3808, Jul. 2015.

[33] R. Tibshirani, "Regression shrinkage and selection via Lasso," J. R. Stat. Soc. Ser. B Methodol., vol. 58, no. 1, pp. 267-288, 1996. 
[34] Z. Tan and A. Nehorai, "Sparse direction of arrival estimation using co-prime arrays with off-grid targets," IEEE Signal Process. Lett., vol. 21, no. 1, pp. 26-29, Jan. 2014.

[35] D.E. Goldberg, Genetic Algorithms in Search, Optimization and Machine Learning, Reading, MA: Addison-Wesley, 1989.

[36] J. Kennedy and R. Eberhart, "Particle swarm optimization," in Proc. IEEE International Conference on Neural Networks, Perth, WA, 1995, pp. 1942-1948.

[37] G.B. Fogel and D.B. Fogel, "Continuous evolutionary programming: Analysis and experiments," Cybern. Syst., vol. 26, pp. 79-90, 1995.

[38] H.P. Schwefel, Evolution and Optimum Seeking, Sixth Generation Computer Technology Series, New York: Wiley, 1995.

[39] E. BouDaher and A. Hoorfar, "Electromagnetic optimization using mixed-parameter and multiobjective covariance matrix adaptation evolution strategy," IEEE Trans. Antennas Propag., vol. 63, no. 4, pp. 1712-1724, Apr. 2015.

[40] E. BouDaher, F. Ahmad, M. Amin, and A. Hoorfar, "DOA estimation with co-prime arrays in the presence of mutual coupling," in Proc. $23^{\text {rd }}$ European Signal Processing Conference, 2015, pp. 2830-2834.

[41] N. Hansen, "A CMA-ES for mixed integer nonlinear optimization," Institut National de Recherche en Informatique et en Automatique, no. 7751, 2011. 
TABLE I

MINIMUM REDUNDANCY ARRAY CONFIGURATIONS

\begin{tabular}{|c|c|}
\hline$N_{A}$ & Positions \\
\hline 4 & {$[0,1,4,6] d_{0}$} \\
\hline 6 & {$[0,1,6,9,11,13] d_{0}$} \\
\hline 8 & {$[0,1,4,10,16,18,21,23] d_{0}$} \\
\hline 10 & {$[0,1,3,6,13,20,27,31,35,36] d_{0}$} \\
\hline
\end{tabular}

TABLE II

NESTED ARRAY CONFIGURATIONS

\begin{tabular}{|c|c|}
\hline$N_{A}$ & Positions \\
\hline 4 & {$[0,1,2,5] d_{0}$} \\
\hline 6 & {$[0,1,2,3,7,11] d_{0}$} \\
\hline 8 & {$[0,1,2,3,4,9,14,19] d_{0}$} \\
\hline 10 & {$[0,1,2,3,4,5,11,17,23,29] d_{0}$} \\
\hline
\end{tabular}

TABLE III

CO-PRIME ARRAY CONFIGURATIONS

\begin{tabular}{|c|c|c|c|}
\hline$N_{A}$ & $\mathrm{M}$ & $\mathrm{N}$ & Positions \\
\hline 4 & 2 & 3 & {$[0,2,3,4] d_{0}$} \\
\hline 6 & 3 & 4 & {$[0,3,4,6,8,9] d_{0}$} \\
\hline 8 & 4 & 5 & {$[0,4,5,8,10,12,15,16] d_{0}$} \\
\hline 10 & 5 & 6 & {$[0,5,6,10,12,15,18,20,24,25] d_{0}$} \\
\hline
\end{tabular}




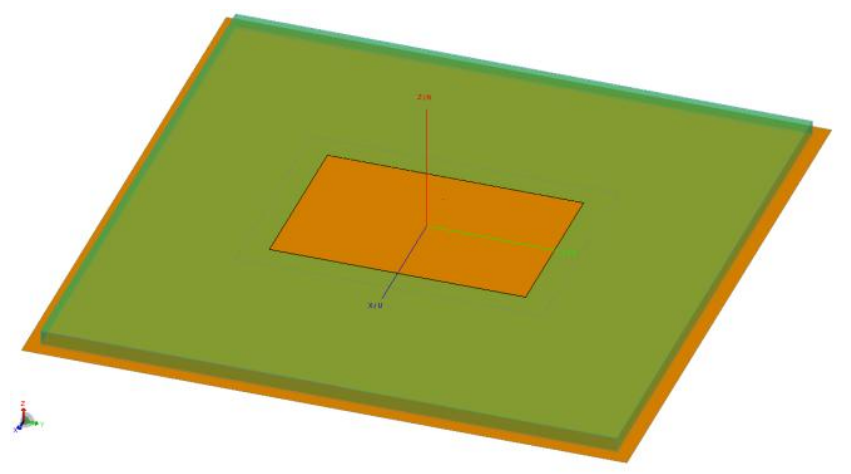

(a)

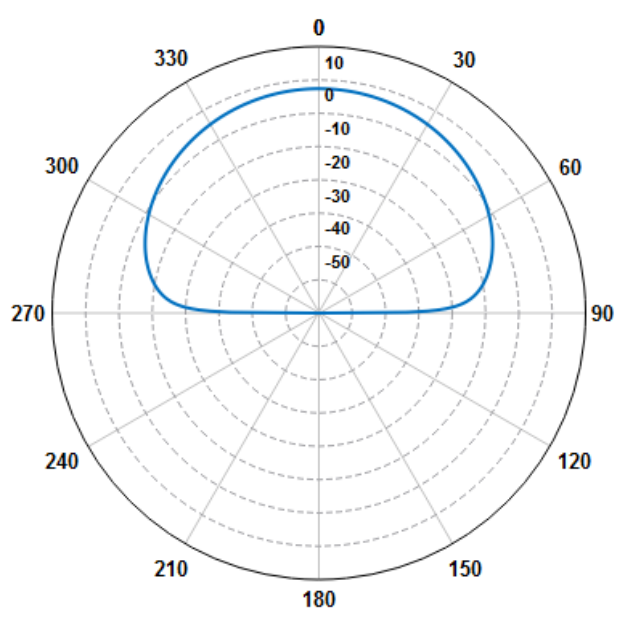

Total Gain [dBi] (Frequency $=3 \mathrm{GHz}$; Phi $=90 \mathrm{deg}$ )

(b)

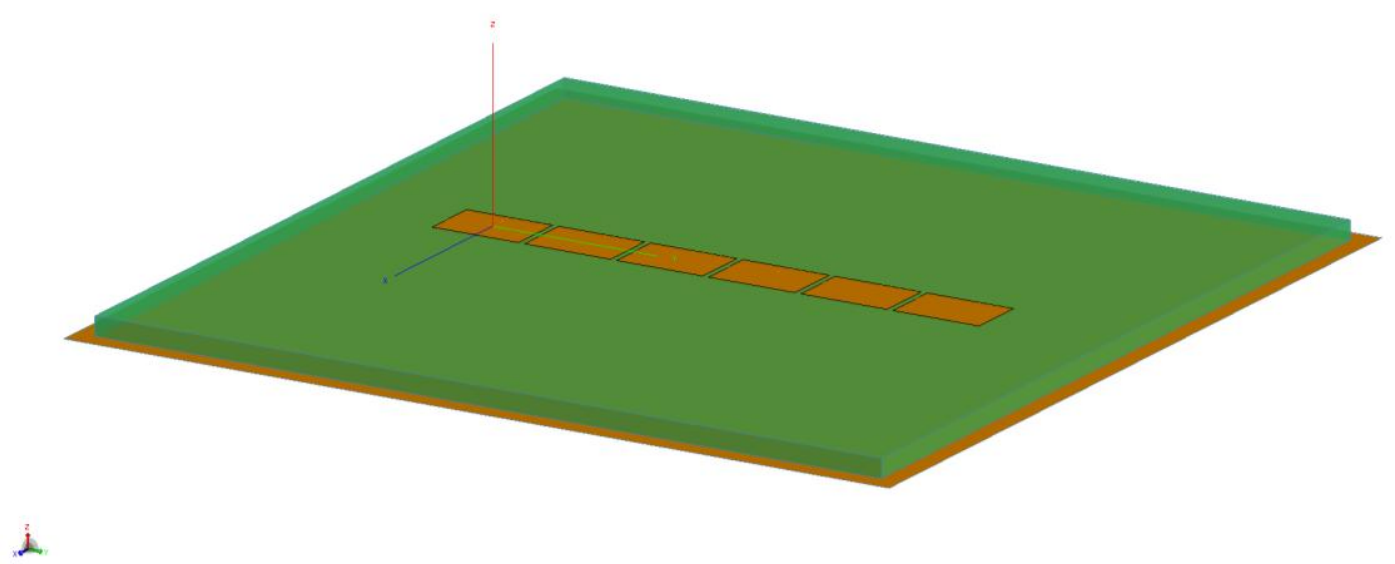

Figure 1. (a) Patch antenna, (b) Gain pattern (dBi) of a single element in isolation, (c) Sixelement uniform linear patch array. 


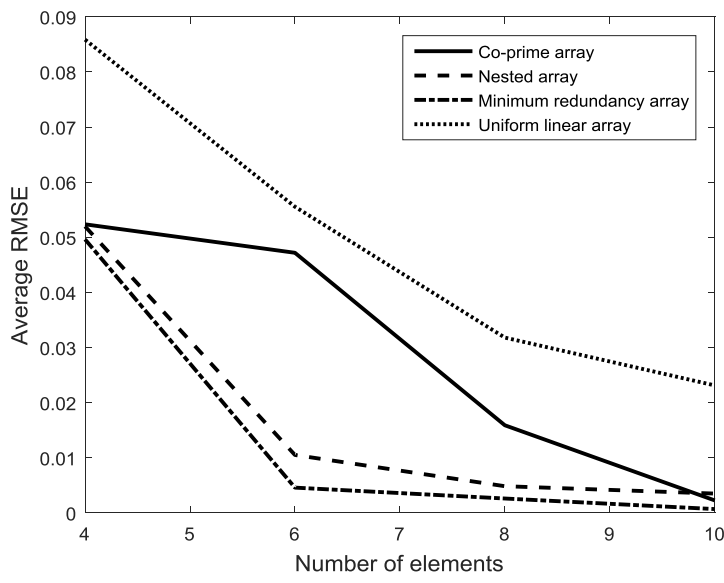

(a)

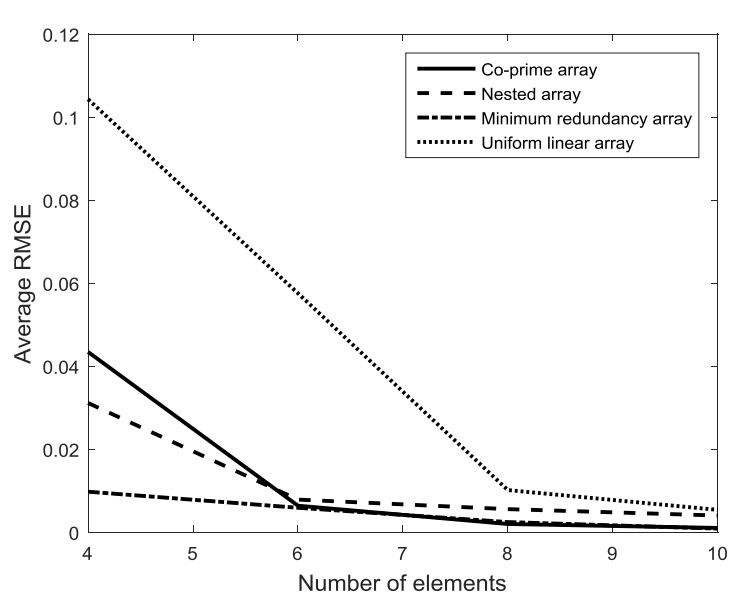

(c)

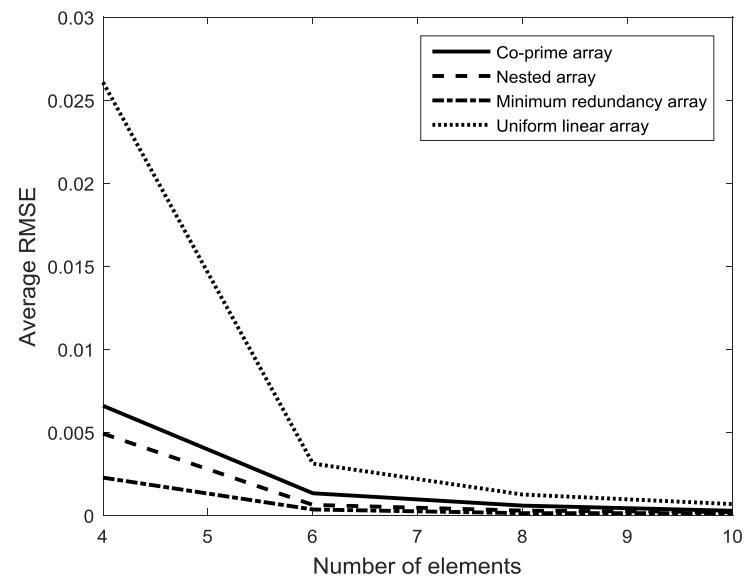

(b)

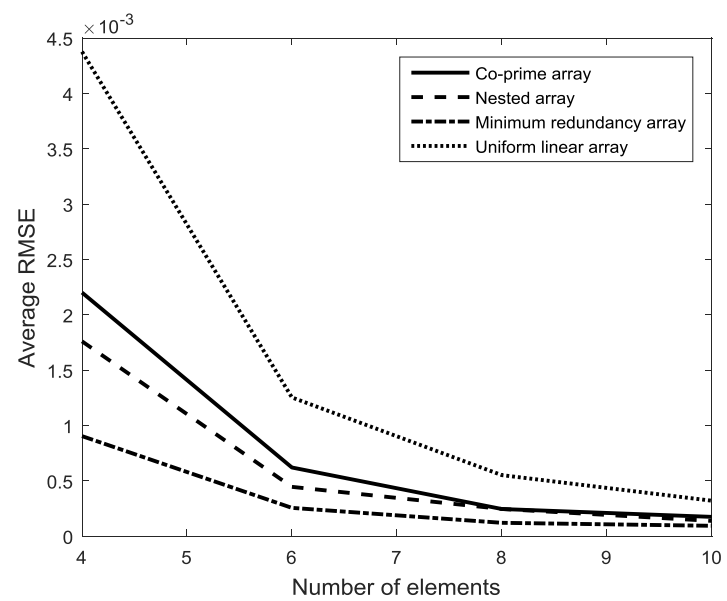

(d)

Figure 2. Dipole arrays: Average RMSE for different array geometries and different number of elements at $\mathrm{SNR}=0 \mathrm{~dB}$, (a) $\Delta u=0.1$ in the presence of mutual coupling, (b) $\Delta u=0.1$ in the absence of mutual coupling, (c) $\Delta u=0.2$ in the presence of mutual coupling, (d) $\Delta u=0.2$ in the absence of mutual coupling. 


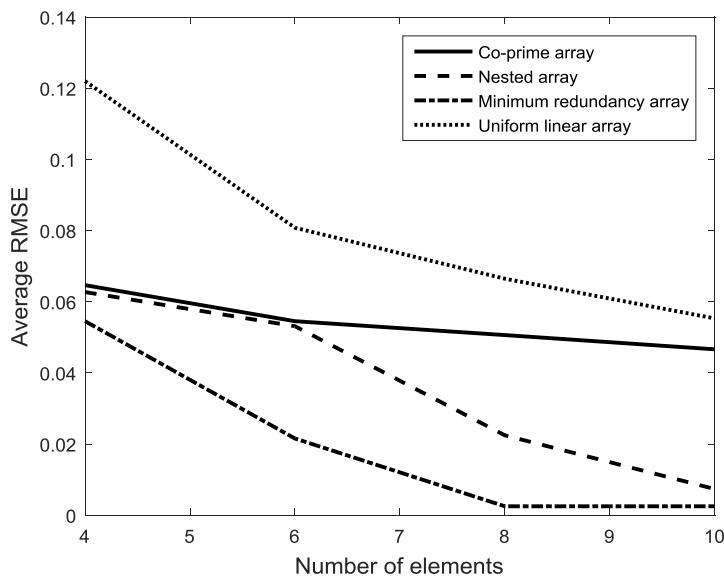

(a)

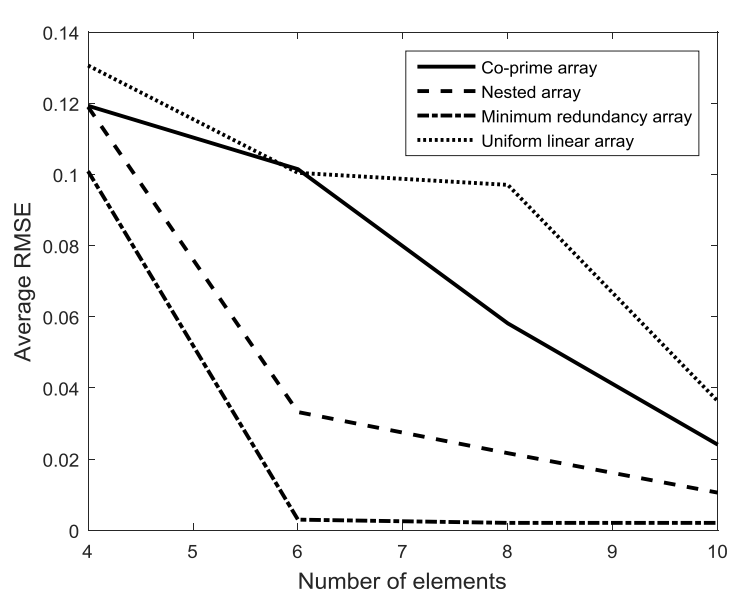

(c)

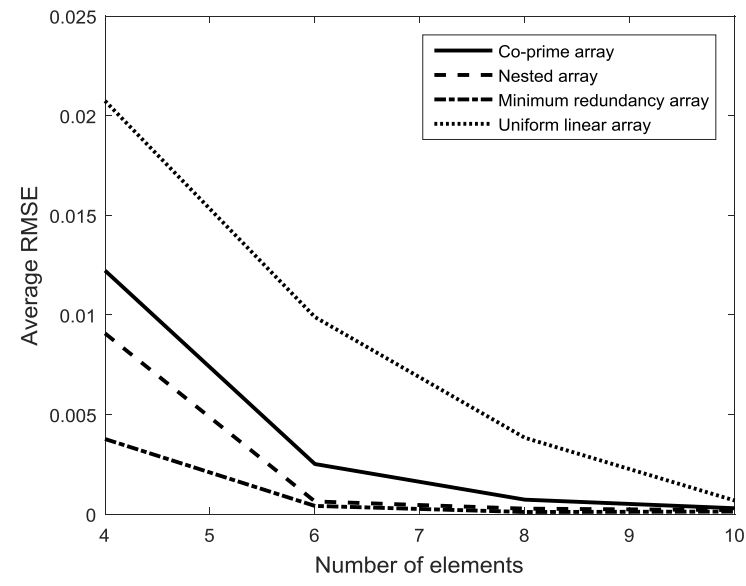

(b)

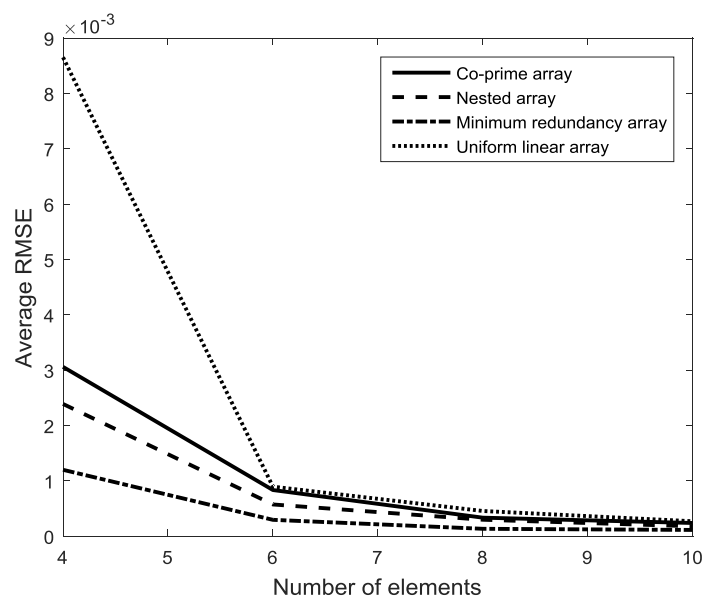

(d)

Figure 3. Microstrip arrays: Average RMSE for different array geometries and different number of elements at $\mathrm{SNR}=0 \mathrm{~dB}$, (a) $\Delta u=0.1$ in the presence of mutual coupling, (b) $\Delta u=0.1$ in the absence of mutual coupling, (c) $\Delta u=0.2$ in the presence of mutual coupling, (d) $\Delta u=0.2$ in the absence of mutual coupling. 


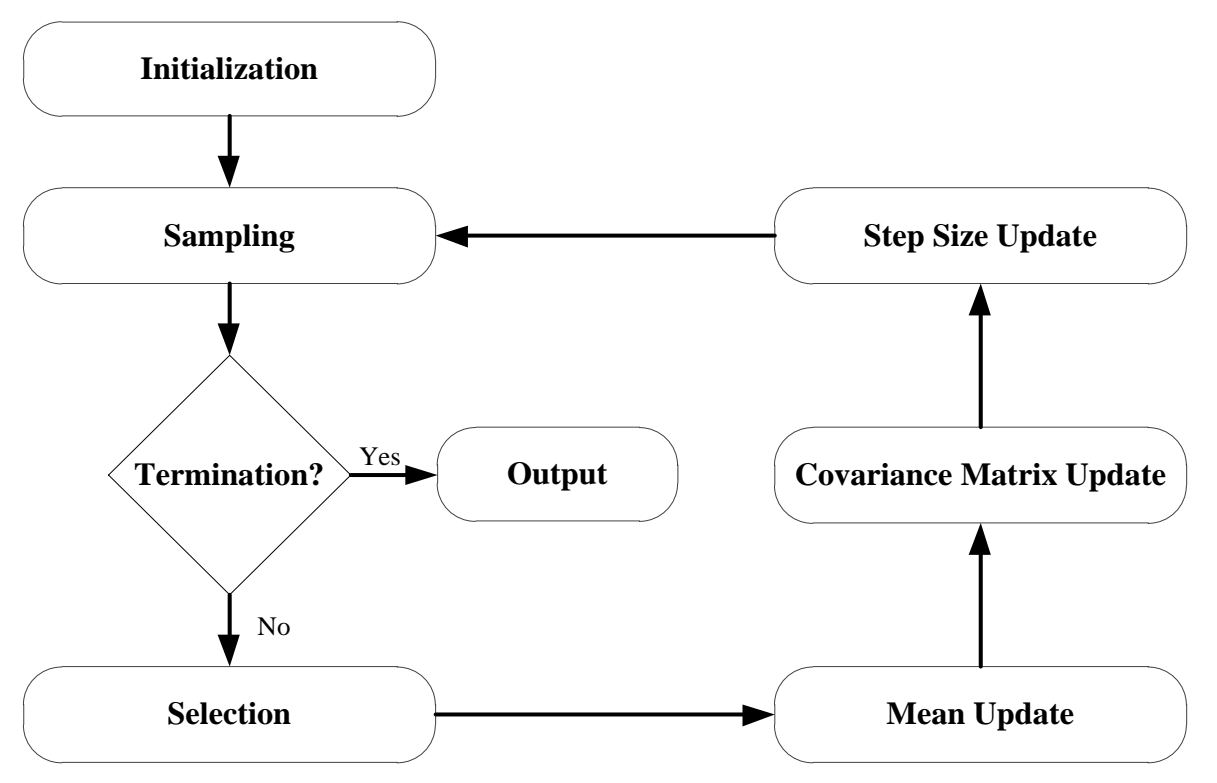

Figure 4. CMA-ES block diagram.

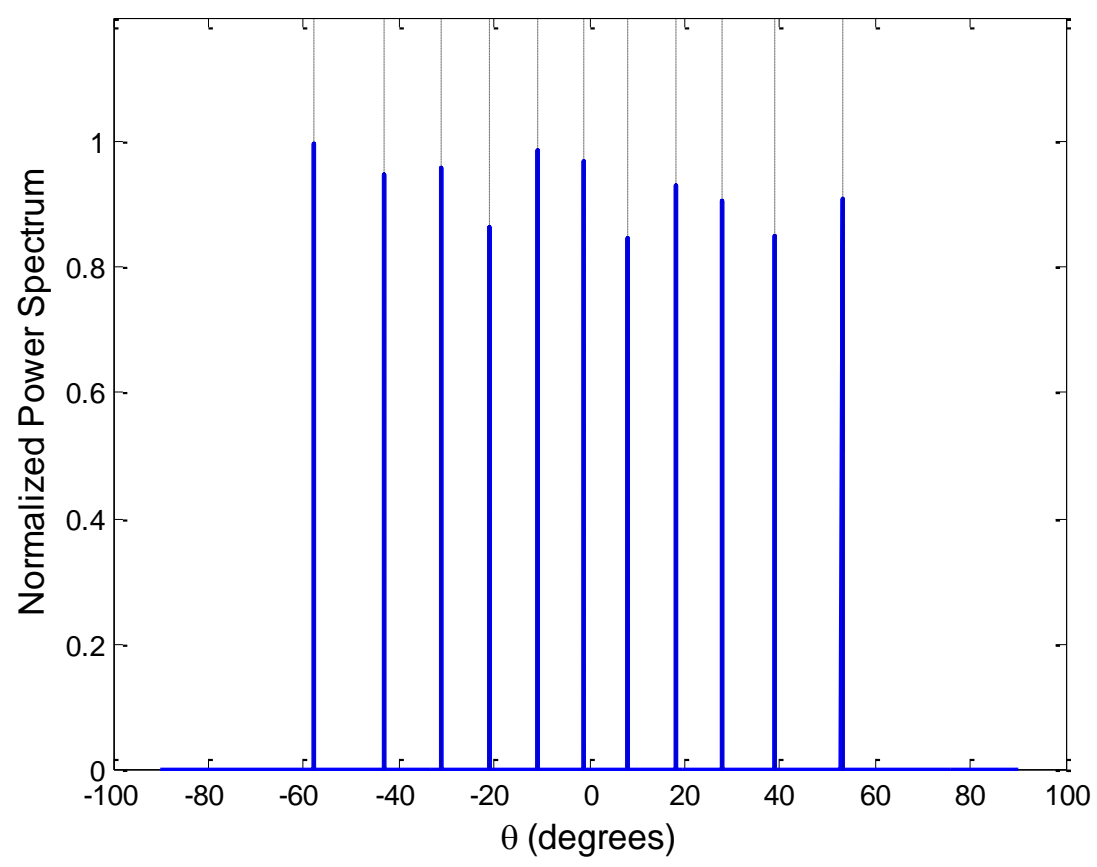

Figure 5. Simultaneous approach estimated spectrum: Six-element nested-array, $D=11$. 


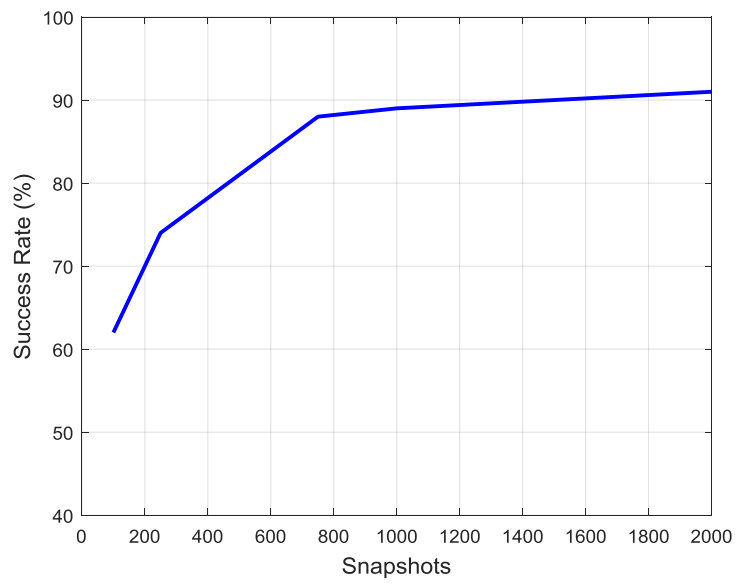

(a)

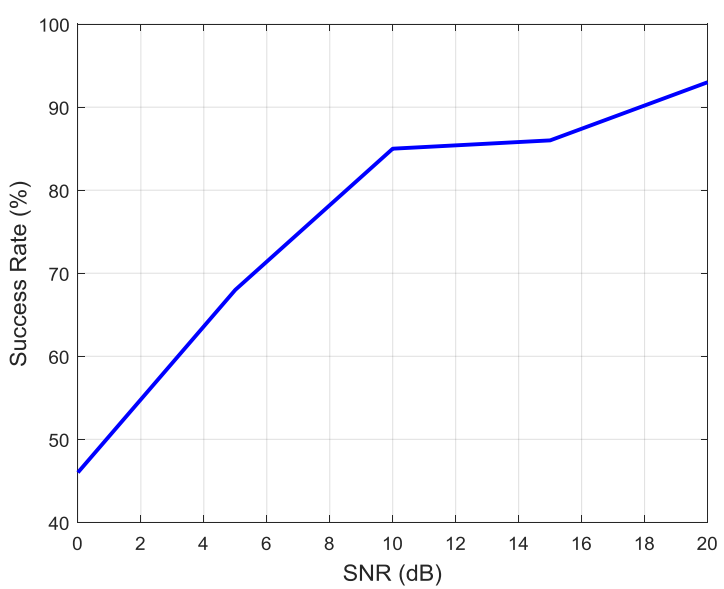

(c)

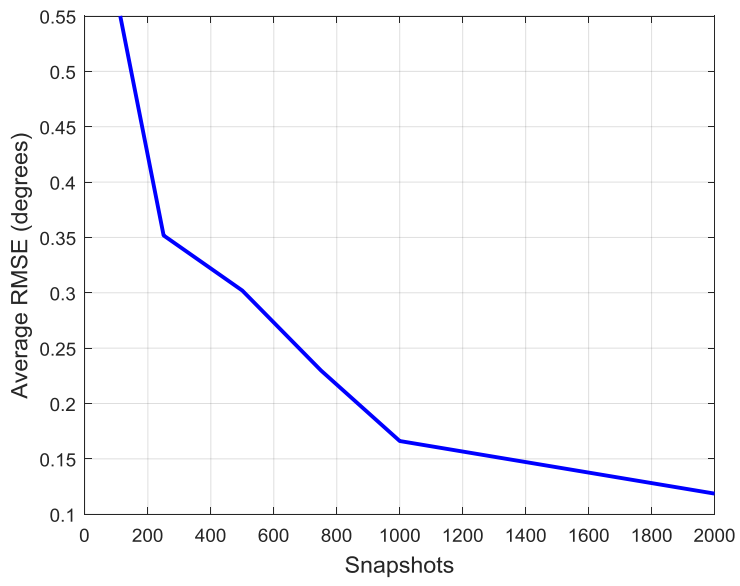

(b)

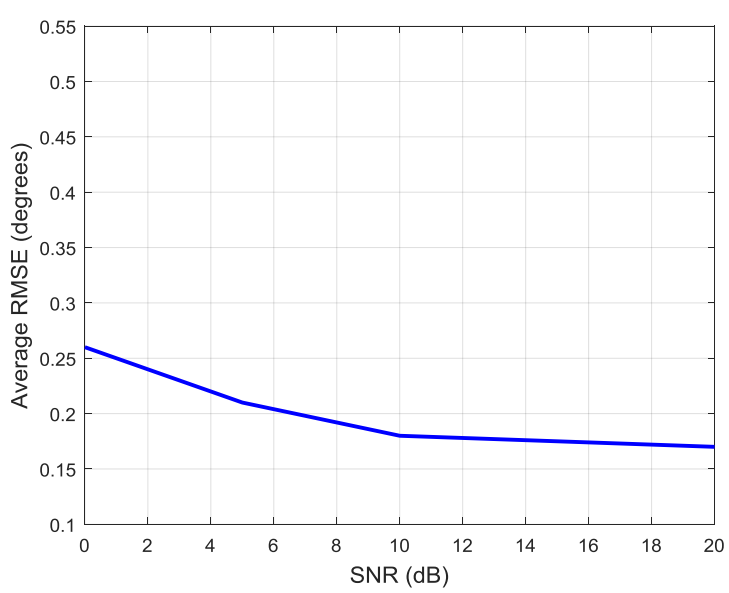

(d)

Figure 6. Simultaneous approach: Six-element nested-array, $D=5$; (a) $\mathrm{SNR}=10 \mathrm{~dB}$, success rate vs. snapshots, (b) SNR $=10 \mathrm{~dB}$, average RMSE vs. snapshots (c) $\mathrm{T}=1000$ snapshots, success rate vs. SNR, (d) T = 1000 snapshots, average RMSE vs. SNR. 


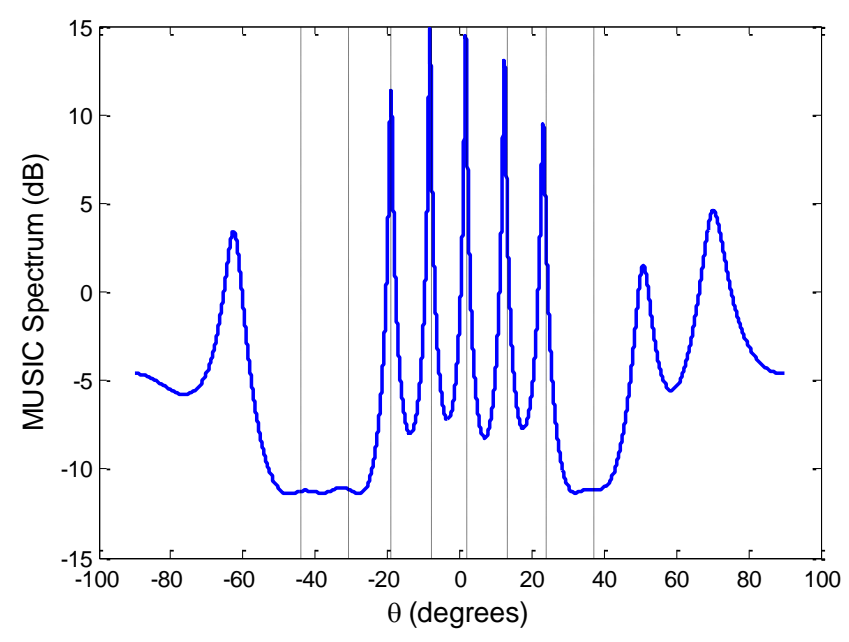

(a)

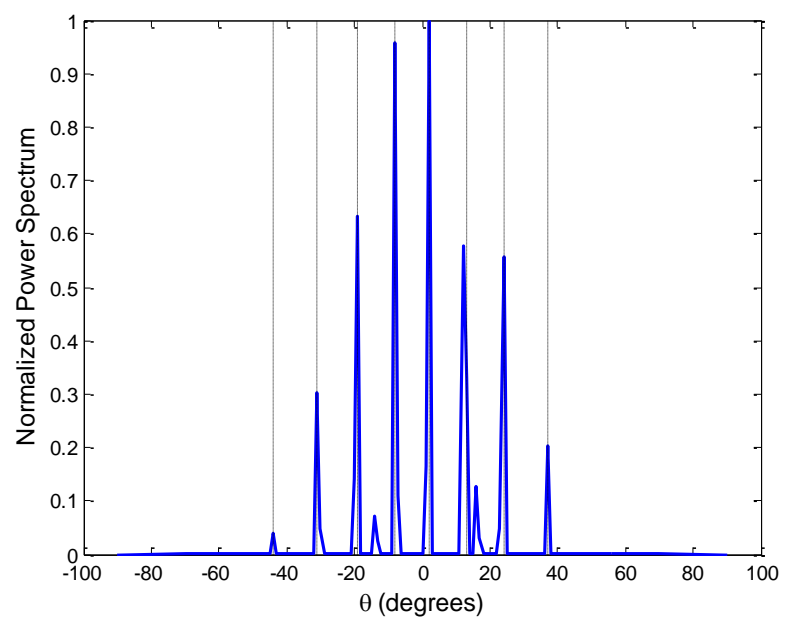

(c)

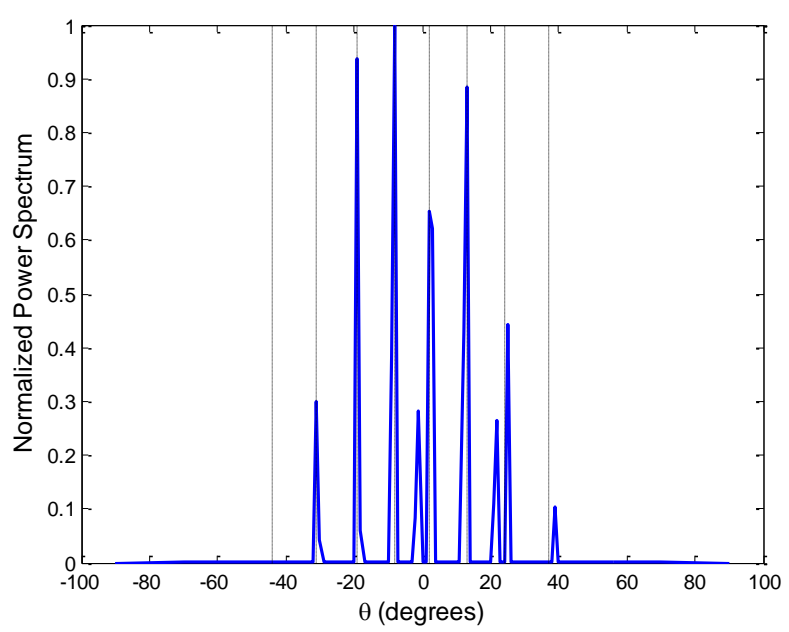

(b)

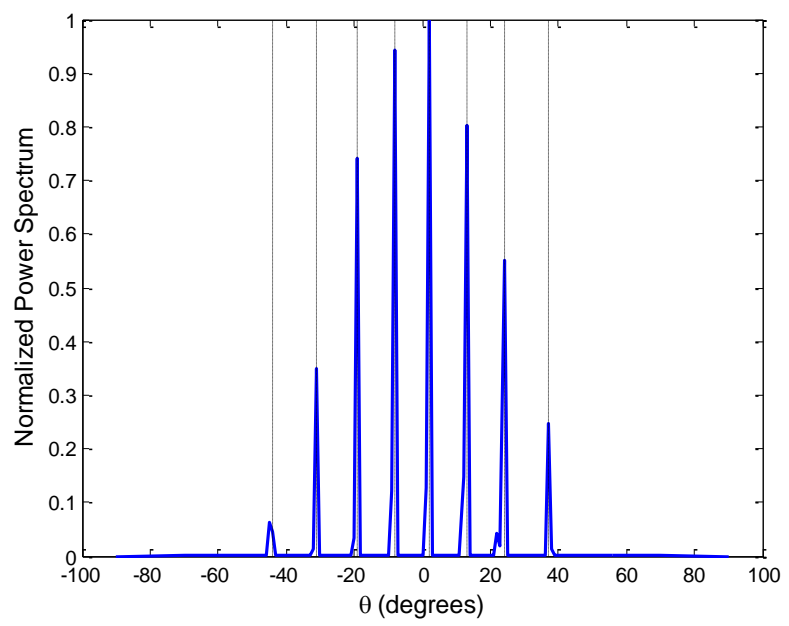

(d)

Figure 7. Six-element microstrip array (a) MUSIC with spatial smoothing without accounting for mutual coupling, Iterative method: (b) initial estimated spectrum, (c) estimated spectrum after first iteration, (d) estimated spectrum after tenth iteration. 


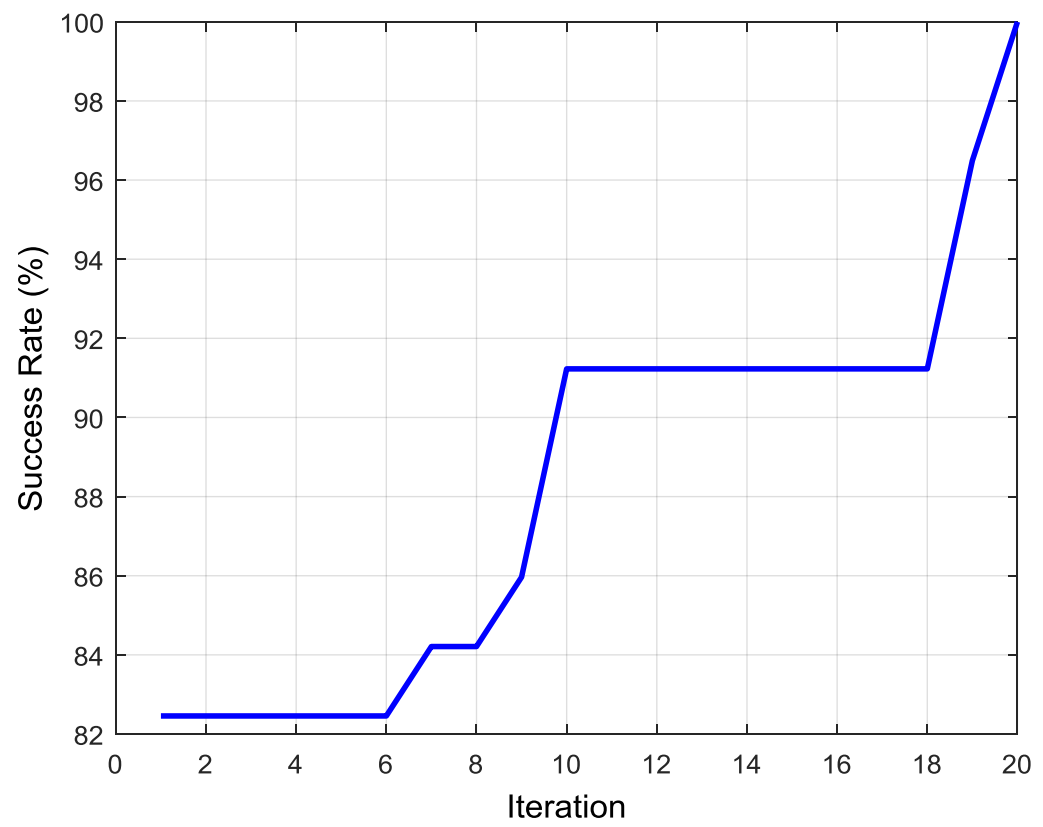

Figure 8. Six-element microstrip array: Success rate vs. iteration number.

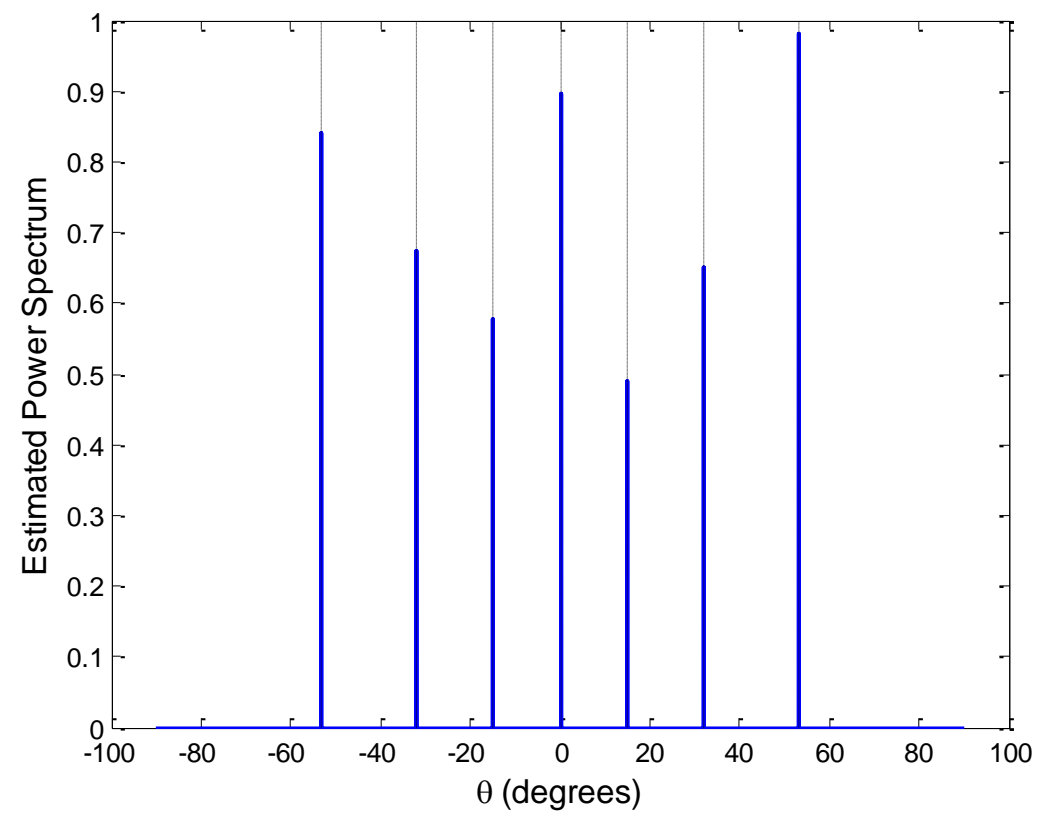

Figure 9. Estimated spectrum: Extended co-prime array with $M=2, N=3, D=7$. 


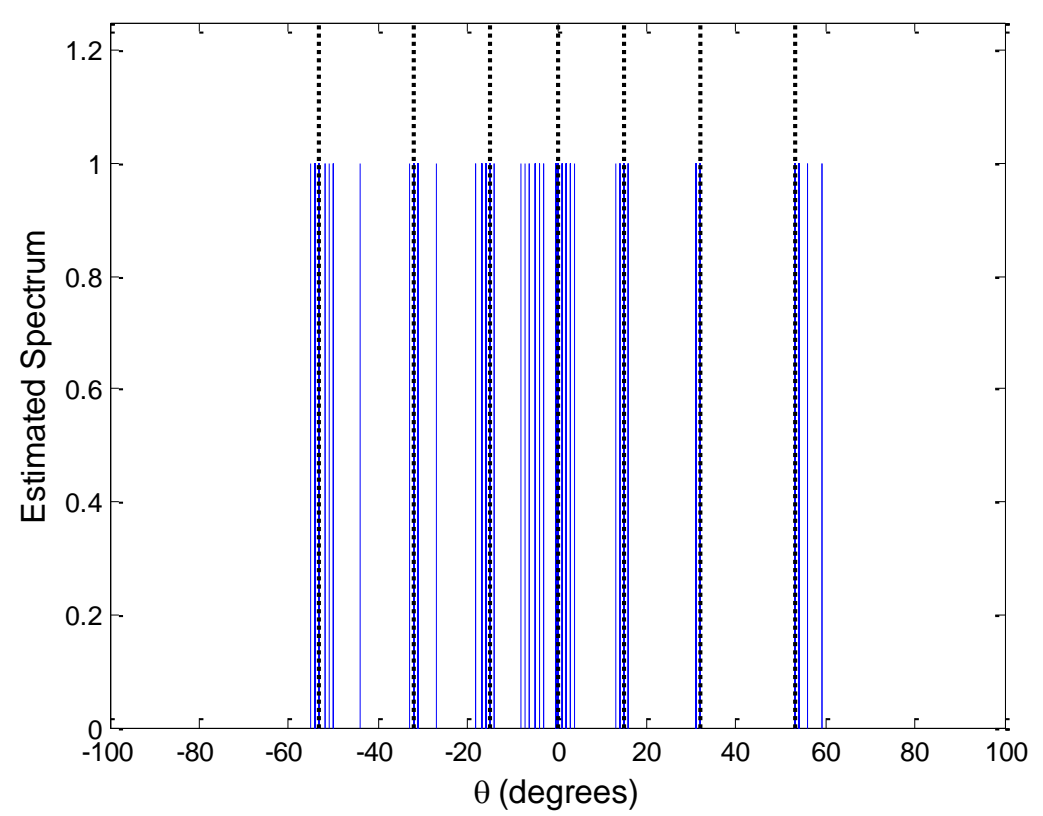

(a)

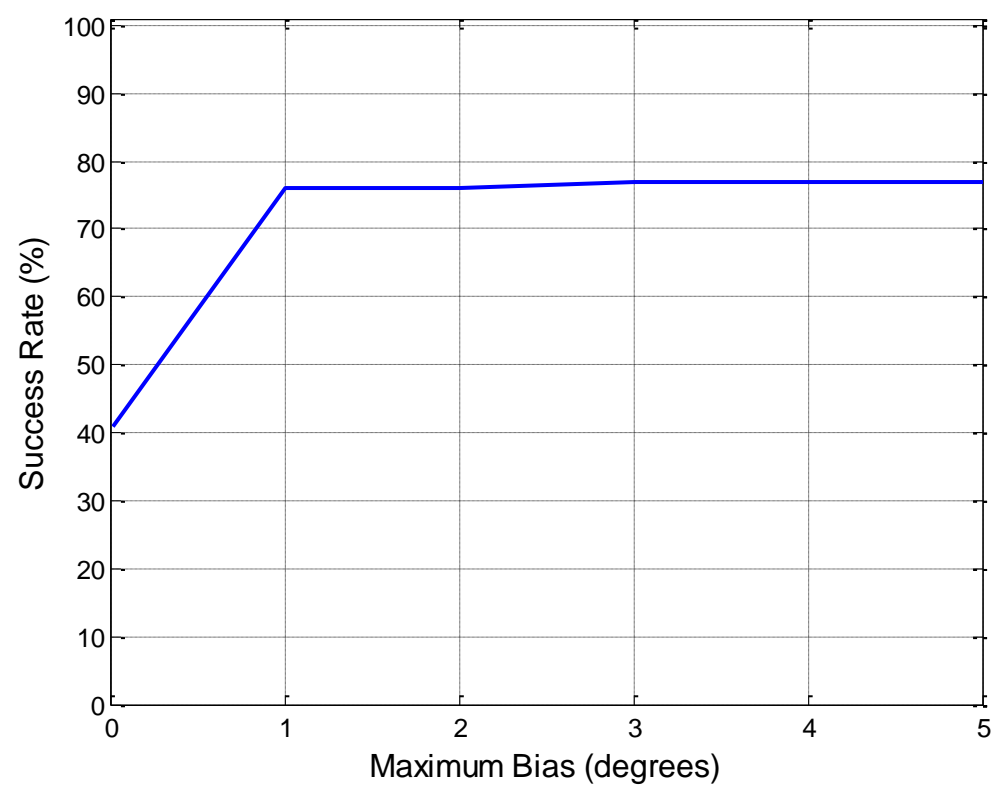

(b)

Figure 10. CMA-ES population size: 1000 with no restarts, (a) Estimated DOAs of 100 the Monte Carlo runs, (b) Success rate of the obtained solutions. 


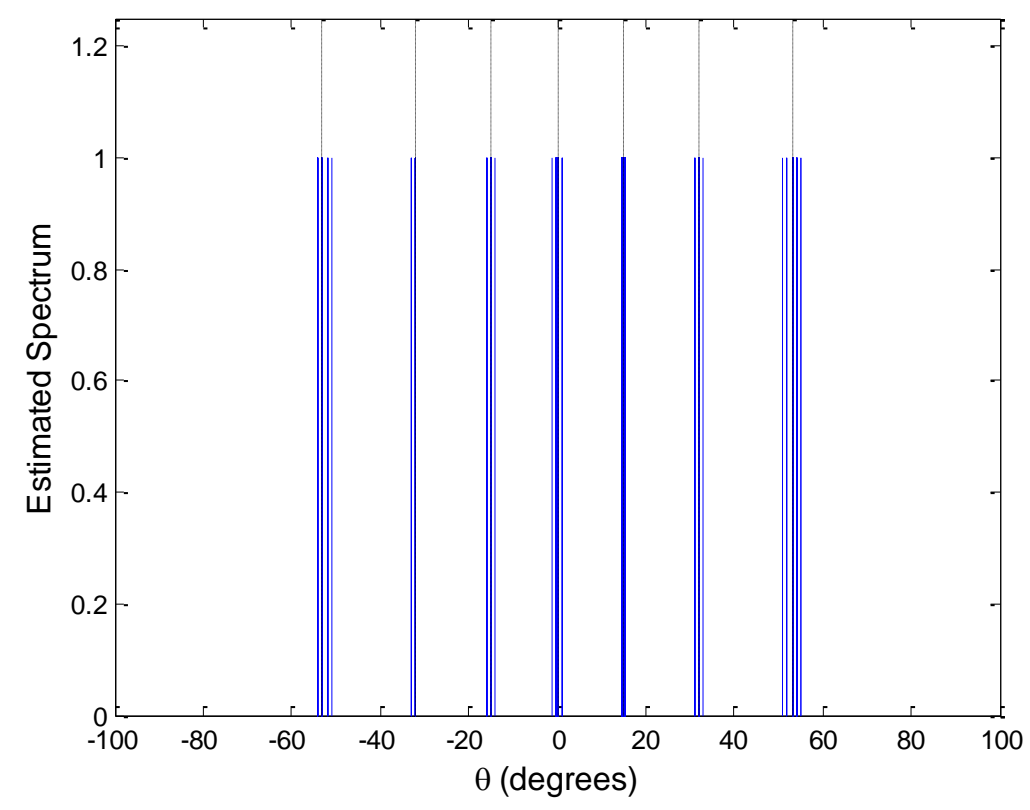

(a)

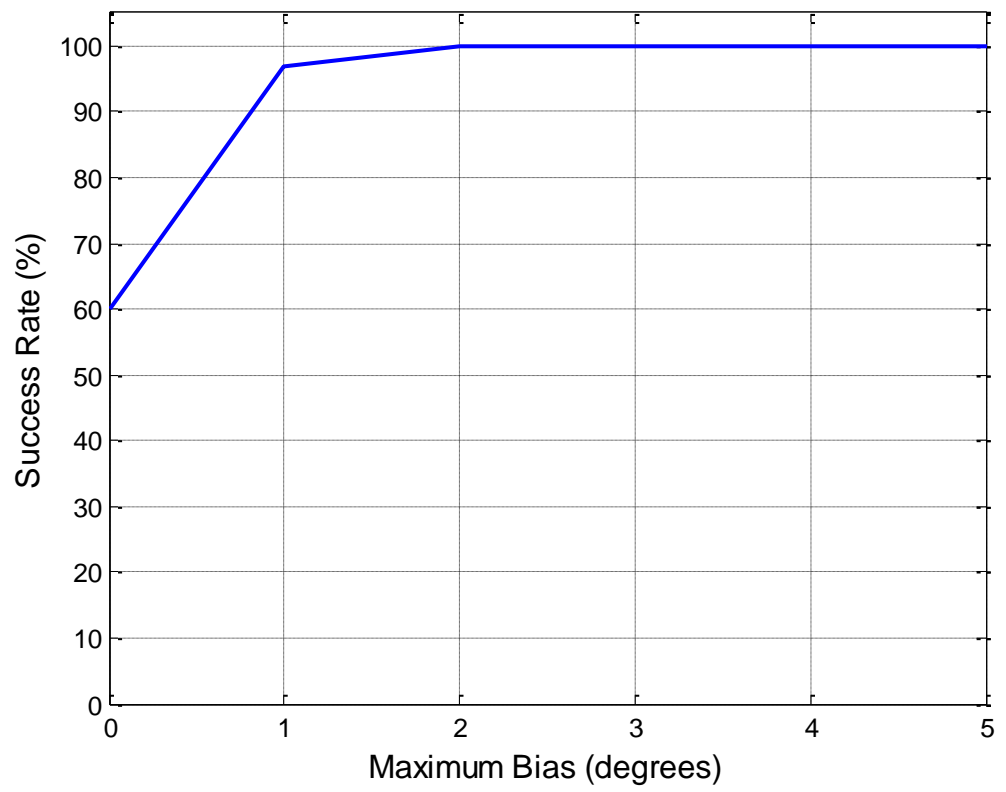

(b)

Figure 11. CMA-ES population size: 5000 with five restarts, (a) Estimated DOAs of 100 the Monte Carlo runs, (b) Success rate of the obtained solutions. 